\title{
Proteomics:A New Diagnostic Horizon In Periodontics.
}

\author{
Dr.Veena A.Patil, Dr.Pradnya.Wagh, Dr.Jignesh.Patel, Dr.B.Bhargavi, \\ Dr.Bibina George \\ H.K.E.S'S S.N.Institute Of Dental Sciences And Research, Kalaburagi.
}

\begin{abstract}
Periodontitis is an inflammatory condition resulting from the interplay between the infectious agents and host factors. Various protein molecules play a vital role in the initiation, progression and severity of periodontal diseases. The study of proteins as biomarkers in periodontal diseases has been highlighted during the last few years. In periodontitis, multiple bacteria derived and host derived mediators expressed in the saliva and gingival crevicular fluid, can be utilized as diagnostic markers for the disease. Another significant development regarding human genes and proteins has been the discovery of potential new drugs for the treatment of periodontal diseases. Therefore the information of the proteins involved in the pathogenesis of periodontal diseases can be utilized for its diagnosis, prevention and treatment.
\end{abstract}

Keywords: Proteome,Periodontitis,Proteins,Biomarkers,Genome.

\section{Introduction}

Periodontal disease is considered as an inflammatory disorder that damages tissues through complex interaction between bacteria and the host defense system. The onset, progression and severity of periodontal disease are mediated by various protein molecules. Periodontal tissues constitutes multi compartmental groups of interacting cells and matrices that render continuous support, attachment, proprioception and physical protection for teeth. ${ }^{[1]}$ The knowledge of various proteins involved in periodontal disease pathogenesis helps in diagnosis, prevention and treatment of periodontal diseases. In periodontology, proteomes - the complete protein pool of an organism, are vital for understanding periodontal ligament physiology and regulation and to diagnose disease related protein markers. Proteomics- the large scale analysis of proteins- has become one of the most important disciplines for gene function characterization, building functional linkages between protein molecules, and to comprehend the mechanisms of biological processes. "PROTEOMICS" is a relatively new field; and is defined as the total protein content of a cell or that of an organism. The term "proteome" a blend of "protein" and "genome" was coined by Marc Wilkins in 1996. ${ }^{[2]}$ Proteomic studies analyses the structure and function of various proteins and the protein-protein synergism of an organism. Any minor defects either in protein structure, its function or alteration in expression pattern can be detected using proteomic techniques.

Recent progress in tissue isolation, protein separation, quantification, sequence analysis and structural interaction using proteomic techniques offers great promise for bringing about a change in periodontal physiology and pathology into the modern era. Hence this review targets at bringing into limelight, the potential proteomic avenues that may be applied to periodontal tissues and the opportunities that may arise in defining the expressed set of proteins in the periodontium.

\section{Rationale of periodontics:}

Apart from analysing the structure and function of biological systems, the objective of proteomics is to analyse the varying proteomes of an organism at different times, in order to

highlight the differences between them. Among the many objectives of proteomics, the discovery of novel protein biomarkers has been a major driving force in the development of proteome characterization methodologies. ${ }^{[3]}$

\section{Types Of Proteomics}

Structural Proteomics: Study of proteomics is based on structural information of total repertoire for three dimensional images for all proteins in an organism. This arises from analysis of unknown proteins such as protein bound ligand or cofactor and is useful for functional description. ${ }^{[1]}$ The identification of all proteins on a genome wide scale, determining their structural-functional relationships, and describing three-dimensional structures are the important hurdles in structural proteomics. ${ }^{[4]}$ Functional and evolutionary protein relation which were not visible at sequence level are now possible with the advent of structural proteomics ${ }^{[5]}$

Interaction Proteomics: The functions of biological systems are dependent on interactions between their components. These interactions are ultimately determined by genetic elements and selection processes. ${ }^{[6]}$ The sequencing of complete genomes provides information on the proteins responsible for cellular regulation. The different technique used for this includes yeast two-hybrid system, microassays, and affinity purification. 
This technology has been used for many different biological systems including, for example, identification of novel matrix metalloproteinase substrates that act to regulate inflammation. ${ }^{[7]}$

Functional Proteomics:Types of proteins that indicate the function of proteins or how they are assembled into the molecular machines and functional networks that regulate cell behaviour ${ }^{[8]}$ determine the functional proteomics. It is "focused to monitor and analyse the spatial and temporal properties of the molecular networks and fluxes involved in the living cells" ${ }^{[9]}$. It concentrates on the following two issues ${ }^{[10]}$ :

(i) elucidation of biological functions of unknown proteins,

(ii) cellular activity at molecular level.

\section{Methods of proteome analysis:}

The tissue and cell complexity in the periodontium require the submission of more global experimental approaches for determining expression profiles ${ }^{[1]}$. Proteomic armamentarium contains a broad array of technical approaches ${ }^{[1]}$. For analysis of dissected periodontal tissues, sections through periodontium or cultured periodontal cells, fractionation of cells, and matrix followed by protein separation are the initial steps to proteomic study. The enumerated methods of proteome analysis are below.

(a) ELISA (Enzyme Linked Immunosorbent Assay) which is a tried and tested method for isolation and quantification of protein.

(b) In 1995, Randall Nelson pioneered the use of immunoassays with mass spectrometry (MSIA). To determine the set of proteins that have undergone posttranslational modification, antibodies can be developedwhich are specific to themodifications and can only recognize certain proteins.

(c) Recently, another approach has been developed called PROTOMAP (Protein Topography and Migration Analysis Platform) which combines Sodium Dodecyl Sulphate Poly Acrylamide Gel Electrophoresis (SDSPAGE) with shotgun proteomics to enable detection of changes in gel migration such as those caused by proteolysis or posttranslational modification ${ }^{[11]}$.

(d) More recent techniques such as matrix-assisted laser desorption/ionization ${ }^{[12]}$ have been employed for rapid determination of proteins in particular mixtures.

(e) For analysis of complex protein mixtures derived from biological samples, two-dimensional polyacrylamide gel electrophoresis ${ }^{[13]}$ remains an important technology.

(f) Nongel based proteome separation techniques to overcome the limitations of two-dimensional electrophoresis while preserving the ability to resolve complex protein and peptide mixtures before mass spectrometry analysis were developed.

(g) Capillary electrophoresis is an alternative to both two dimensional electrophoresis for protein separation and to chromatography for peptide separation.

Mass spectrometer-based proteomic analysis is now being used more frequently in studies of interest to dental scientists including, for example, the analysis of Streptococcus mutans and the analysis of osteoblastic differentiation ${ }^{[14,15] .}$ The sequence of mass spectrometric analysis of an unknown mixture of proteins includes, first, the separation of proteins from the biological sample, digestion of the proteins, separation of the peptides, and then analysis of proteins by mass spectrometry and sequence analysis ${ }^{[1]}$. Mass spectrometers have improved ability to detect and characterize the amount of protein in biological samples. Still a major challenge is to determine how the complement of expressed cellular proteins - the proteome-is organized into functional, higher-order networks and to develop global protein-protein interaction networks on a cellular or tissue level ${ }^{[16]}$.The following flow chart illustrates the major steps from the separation of the fractionated proteins till the determination of its sequence analysis ${ }^{[1]}$ (Figure 1).

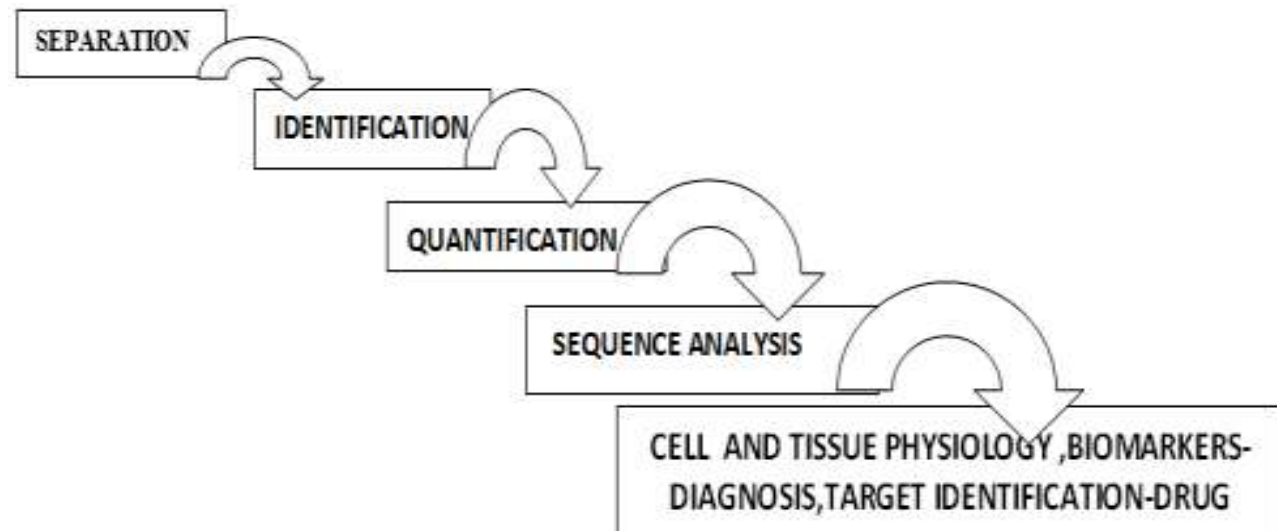

Figure 1: The Major Steps Of Separation To Sequence-Analysis Of The Fractionated Proteins. 


\section{Proteomics and dentistry:}

Proteomics is a relatively new postgenomic science with tremendous potential and has become one of the most important research frontiers in modern dentistry. Proteomics has quickly become one of the most exciting research frontiers in modern dentistry. The two primary areas which dental proteomics have really shown are salivary diagnostics i.e. oral fluid diagnostics or oral fluid biomarkers ${ }^{[17,18]}$ and proteomics of bone and enamel structures, especially dental enamel ${ }^{[19]}$.

Human saliva contains proteins that can be informative for disease detection and surveillance of oral health. Comprehensive analysis and identification of the proteomic contents in human whole and ductal saliva is a necessary first step toward the discovery of saliva protein markers for human disease detection in particular for oral cancer and Sjogren's syndrome. ${ }^{[20,21]}$

\section{Proteomics in periodontal ligament fibroblasts:}

To understand PDL physiology and disease related protein markers, the analysis of the entire complement of PDL fibroblast proteome is of utmost importance. Although immunological methods have been tried to study the protein expression of PDL fibroblast, but this is limited to only previously identified proteins for which antibodies are available. Characterization of periodontal ligament (PDL) fibroblast proteome is an important tool for understanding PDL physiology and regulation and for identifying disease-related protein markers. PDLfibroblast protein expression has been studied using immunological methods, although this technique is limited to previously identified proteins for which specific antibodies are available. A total of 117 proteins have been identified from PDL fibroblasts which can serve as a reference map for future clinical studies as well as basic research. ${ }^{[22]}$

\section{Proteomic biomarkers:}

Periodontal disease is a bacteria-induced chronic inflammatory disease affecting the soft and hard supporting structures encompassing the teeth. ${ }^{[2]}$ Assessment of periodontal disease is based on clinical parameters and radiographs. Though efficient, these traditional techniques are limited, as the current status of the disease alone can be determined. They do not have the capacity to diagnose susceptible patients who are at risk for disease progression. Development in the use of oral fluids as biological samples for measuring the present disease state, has made saliva and other oral-based fluids the vanguard of technology. Oral fluids comprises of local and systemic mediators of periodontal disease, ${ }^{[25]}$ which comprises of microbial, host response, and markers specific for bone resorption.

Though most biomarkers in oral fluids exhibit inflammatory mediators, specific collagen downgrading and bone turn overrelated molecules have become apparent measures of periodontal disease. In oral diagnostics, it has been a great challenge to determine biomarkers for screening and predicting the early onset of disease or evaluating the disease activity and the efficacy of therapy. An oral diagnostic tool should provide relevant information for differential diagnosis, localization of disease and severity of infection. It should serve as a basis for treatment planning and act as a means of assessing the effectiveness of therapy. Thus keeping these criterias in mind researchers have made an effort to understand the underlying science of the disease, with an aim to find improved ways to diagnose and treat the disease before any serious outcome. Human saliva in oral diagnostics is of great importance and researchers are concentrating on it. Saliva is considered as an important Periodontal diagnostic tool since variable amounts of blood, serum and its products, GCF, epithelial cells as well as immune cells, microorganisms, products obtained from bacterial degradation, lipopolyscaaharides, bronchial products and other foreign substances are present in whole saliva. ${ }^{[26]}$ Saliva, has high potential for the surveillance of general health and disease. It represents a promising diagnostic fluid for the screening of various oral diseases. It is a fluid that comprises of constituents of exocrine glands and gingival crevicular fluid (GCF). ${ }^{[2]}$ Moreover, saliva is effortlessly available and easily collected without any cumbersome procedure. Matrix Metalloproteinases (MMP 2 and 9), Immunoglobulin (Ig), Esterases, Lysozyme, Lactoferrin levels in saliva are valuable for predicting the progression of periodontitis. Cytokines like C- reactive protein, pentraxin-3, TNF, and other interleukins which are involved in disease pathogenesis are essential for diagnosing periodontal diseases. ${ }^{[3]}$

Apart from this, numerous proteomic markers, like acid phosphatase, alkaline phosphatase, [27] histatins, kallikreins, cystatins, kininogens, aminopeptidases, glucosidase, galactosidase and glucuronidase, and various bone remodelling proteins (Osteopontin, Osteonectin, Osteocalcin) are noted in the diagnosis of periodontal disease. ${ }^{[24]}$ Apart from periodontal diseases, salivary defence systems which comprises of salivary proteins play a significant and vital role in maintaining the health of the oral cavity and preventing caries. ${ }^{[28]}$ Significant amount of salivary phosphopeptides (PRP1/3, histatin-1 and statherin) were associated with the absence of dental caries, affirming the value of these peptides in nurturing tooth integrity. ${ }^{[17]}$ 


\section{Proteomics And Periodontal Pathogens:}

The oral environment contains various colonies of microorganisms comprising of bacteria, fungi, protozoa, and viruses. Oral ecology studies have reviewed the complexity of the interactions that these microorganisms have with their host in both health and disease. In spite of this, dental caries and periodontal diseases are still worldwide oral diseases, resulting in a high level of morbidity among humans. ${ }^{[29]}$ Proteomics offers a new approach to comprehending these holistic changes, as oral micro-organisms adapt to environmental change within their habitats in the mouth.Various microorganisms are present in subgingival plaque, of which only few play a etiological role in the pathogenesis of periodontal diseases in the susceptible host. Specific microorganisms involved in the periodontal pathogenesis are Tannerella forsythia, Porphyromonas gingivalis, Treponema denticola, and Aggregatibacter actinomycetemcomitans. [26] BANA activity (benzoylDLargininenaphthylamide) was exhibited by the members of the "red complex" of periodontal pathogens (T. forsythia, P. gingivalis, and T. denticola) are strongly correlated with periodontal activity. ${ }^{[31]}$

\section{Proteomics And Tissue Engineering:}

Tissue engineering has evolved in recent years, as efficient means for treating various pathological conditions. This scientific knowledge mainly constitutes stem cell procurement, storage, differentiation as well as transplantation which is performed by using specific biomarkers such as proteins. However, the absence of knowledge regarding these prospective markers for stem cells and their specific differentiation remains a considerable limitation for these applications. ${ }^{[29]}$ In future, the proteomic and transcriptomic analyses may pave way to obtain new and hopefully fundamental insights into the protein expression, and cellular biology of mesenchymal stem cell. ${ }^{[32]}$

\section{Salivary Proteomics For Periodontitis:}

Saliva is considered as an important Periodontal diagnostic tool since variable amounts of blood, serum, serum products, GCF, electrolytes, epithelial and immune cells, microorganisms, bacterial degradation products, lipopolyscaaharides, bronchial products and other foreign substances are present in whole saliva. Matrix Metalloproteinases (MMP 2, 39), Immunoglobulin (Ig), Esterases, Lysozyme, Lactoferrin levels in saliva are valuable for predicting the progression of periodontitis. Numerous other salivary proteases have also been used as diagnostics biomarkers. Various cytokines like C- reactive protein, pentraxin-3, TNF, various other interleukins which are involved in its pathogenesis have come handy in diagnosing periodontal diseases. ${ }^{[24]}$

Young-Jin Choi et al studied the GCF in healthy individuals and Periodontitis patients to study biomarkers. He identified azurocidin in the GCF, but not in the saliva, as an upregulated protein in the periodontitis patients. He concluded that azurocidin could be a potential biomarker candidate for the early detection of inflammatory periodontal destruction by gingivitis and some chronic periodontitis. Azurocidin may have an inhibitory role in osteoclast differentiation and, thus, a protective role in alveolar bone loss during the early stages of periodontitis. ${ }^{[33]}$ Melissa M. Grant et al studied the 21-day experimental gingivitis model. The model was designed to enable the study of both the induction and resolution of inflammation. Across the course of experimentally induced gingivitis, He identified 16 bacterial and 186 human proteins. Although abundances of the bacterial proteins identified did not vary temporally, Fusobacterium outer membrane proteins were detected. ${ }^{[34]}$

\section{Proteomics And Stem Cell Research:}

Large scale mesenchymal stem (MSC) cell proteome analyses have been emphasized in recent MSC research. A review by Hye Won park presents an expandable list of MSC proteins which will function as a starting point for the generation of a comprehensive reference map of their proteome. This proteomic and transcriptomic analyses may allow us to obtain new and hopefully fundamental insights into the protein expression, regulation, and cellular biology of MSC. ${ }^{[35]}$

\section{Clinical Applications:}

The use of rapid point-of-care oral diagnostics will greatly advance periodontal surveillance and disease diagnosis over the coming years. ${ }^{[38]}$ Novel technologies such as lab-on-a-chip and microfluidic devices have the potential to manage complex oral fluids such as saliva and gingival crevicular fluid, and helps in determining the patient's periodontal disease-risk profile, current disease activity and response to therapeutic interventions. This approach should acts like a catalyst for clinical decision making and monitoring of episodic disease progression in a chronic infectious disease such as periodontitis. While the future of periodontal disease diagnosis using salivary diagnostics looks encouraging, hurdles to these approaches may be encountered in the clinical setting. These novel periodontal diagnostic technologies need to be validated \& benchmarked with 
existing gold standards of disease, such as alveolar bone levels and clinical attachment levels, in large patient populations.

\section{The Future Of Proteomics : \\ Development Of Biomarkers :}

The two main research frontiers for application of proteomics in dentistry are salivary diagnostics, or oral fluid biomarkers, and proteomics of bone and enamel. While saliva is accessible and its collection is totally noninvasive, its use in clinical diagnostics has only recently been demonstrated. One team of researchers at UCLA, and others, has shown that oral fluid harbors the same composition of disease biomarkers as blood, but in smaller quantities. These scientists have developed, with support of the National Institute of Dental and Craniofacial Research, a molecular sensor that provides the basis for future development of the "Oral Fluid NanoSensor Test (OFNASET)." OFNASET is predicted to be a handheld and easy-to-use instrument that clinicians can use to rapidly detect complex salivary protein and nucleic acid targets. The result will be the ability to clinically detect oral cancer before oral signs and symptoms. ${ }^{[36]}$

\section{Customized drugs :}

One of the most promising developments to come from the study of human genes and proteins has been the identification of potential new drugs for the treatment of disease. This relies on genome and proteome information to identify proteins associated with a disease, which computer software can then use as targets for new drugs. For example, if a certain protein is implicated in a disease, its 3D structure provides the information to design drugs to interfere with the action of the protein. A molecule that fits the active site of an enzyme, but cannot be released by the enzyme, will inactivate the enzyme. This is the basis of new drug-discovery tools, which aim to find new drugs to inactivate proteins involved in disease. As genetic differences among individuals are found, researchers expect to use these techniques to develop personalized drugs that are more effective for the individual.

\section{Computational Method :}

A computer technique which attempts to fit millions of small molecules to the three-dimensional structure of a protein is called "virtual ligand screening". The computer rates the quality of the fit to various sites in the protein, with the goal of either enhancing or disabling the function of the protein, depending on its function in the cell. Agood example of this is the identification of new drugs to target and inactivate the HIV-1 protease. The HIV-1 protease is an enzyme that cleaves a very large HIV protein into smaller, functional proteins. The virus cannot survive without this enzyme; therefore, it could be one of the most effective protein targets for killing HIV. ${ }^{[37]}$

\section{Limitation}

As protein expression and post-translational modifications are dynamic processes, particularly in the periodontium, identification and quantification of proteins alone are not sufficient to understand functional changes. New technologies will be needed to enable combinations of metabolic labeling and identification as well as quantification and measurement of synthesis rates. Also Proteomics experiments conducted in one laboratory are not easily reproduced in another.

\section{Conclusion}

Proteomics is a remarkable tool that can revolutionise the treatment of oral disease in the context of identifying the risk factors, prompt diagnosis, prevention and control, lead to changes in treatment philosophy in all aspects of dental sciences. Understanding the interaction networks requires systematization and analysis of a huge amount of information emerging from experimental studies. In this context, proteomics is a promising tool to change the practice

of dentistry. The use of proteomics and gene expression will advance the diagnosis and treatment of various oral pathological conditions. With the future of proteomics still remaining in the womb of time, further research is required to investigate deeper regarding the application and integration of this new field. Further, proteins being an universal component of all biological functions of the body, the scope of proteomics in health and especially the field of periodontology is expected to scale greater heights. In periodontium, many but not all expressed proteins are tissue-specific and the function of various proteins is modulated by multiple factors, including interactions with other proteins and modifications arising from attached phosphates, sulfates, carbohydrates, and lipids. Current proteomics analyses have the capacity to provide new insights into the repertoire of expressed proteins and some inkling of their interactions, at a more global level than previously considered. An important challenge that needs to be met by research workers in periodontology is to embrace proteomics approaches when appropriate, and start to apply them to critical, unresolved questions such as the 
biological basis for the heterogeneity in gingival, bone, and cementum cell populations.More reviews incorporating other markers and advances in salivary diagnostics are warranted.

\section{References}

[1]. Mcculloch C A. Proteomics for the periodontium: current strategies and future promise. Periodontol 2000.2006; 40: 173-183.

[2]. Marc R. Wilkins. From Proteins to Proteomes: Large Scale Protein Identification by Two- Dimensional Electrophoresis and Arnino Acid Analysis. Nat Bio. 1996; 14 (1): 61-65.

[3]. Lamba M, Singh A, Jithendra KD, Sinha A. Proteomics- a new era of modernization in periodontics. IJRID. 2015; 5(4):48-56.

[4]. F. Yakunin, A. A. Yee, A. Savchenko, A. M. Edwards, and C. H. Arrowsmith, "Structural proteomics: a tool for genome annotation," Current Opinion in Chemical Biology, vol. 8, no. 1, pp. 42-48, 2004

[5]. H.-L. Liu and J.-P. Hsu, "Recent developments in structural proteomics for protein structure determination," Proteomics, vol. 5, no. 8, pp. 2056-2068, 2005.

[6]. G. Cesareni, A. Ceol, C. Gavrila, L. M. Palazzi, M. Persico, and M. V. Schneider, “Comparative interactomics,” FEBS Letters, vol. 579, no. 8, pp. 1828-1833, 2005.

[7]. G. A.McQuibban, J.-H. Gong, E. M. Tam, C. A. G.McCulloch, I. Clark-Lewis, and C.M. Overall, "Inflammation dampened by gelatinase a cleavage of monocyte chemoattractant protein-3," Science, vol. 289, no. 5482, pp. 1202-1206, 2000.

[8]. T. Pawson and P. Nash, "Assembly of cell regulatory systems through protein interaction domains," Science, vol. 300, no. 5618, pp. 445-452, 2003.

[9]. J. Godovac-Zimmermann and L. R. Brown, "Perspectives for mass spectrumetry and functional proteomics," Mass Spectrometry Reviews, vol. 20, no. 1, pp. 1-57, 2001.

[10]. M. Monti, S. Orr’u, D. Pagnozzi, and P. Pucci, "Functional proteomics," Clinica Chimica Acta, vol. 357, no. 2, pp. 140-150, 2005

[11]. M. M. Dix, G. M. Simon, and B. F. Cravatt, "Global mapping of the topography and magnitude of proteolytic events in apoptosis," Cell, vol. 134, no. 4, pp. 679-691, 2008.

[12]. R. Klopfleisch, P. Klose, C.Weise et al., "Proteome of metastatic canine mammary carcinomas: similarities to and differences from human breast cancer," Journal of Proteome Research, vol. 9, no. 12, pp. 6380-6391, 2010.

[13]. P. H. O'Farrell, "High resolution two-dimensional electrophoresis of proteins," The Journal of Biological Chemistry, vol. 250, pp. 4007-4021, 1975.

[14]. C. L. Len, S. J. Cordwell, D. W. S. Harty, and N. A. Jacques, "Cellular and extracellular proteome analysis of Streptococcus mutans grown in a chemostat," Proteomics, vol. 3, no. 5, pp. 627-646, 2003

[15]. K. A. Conrads, L.-R. Yu, D. A. Lucas et al., "Quantitative proteomic analysis of inorganic phosphate-induced murine MC3T3-E1 osteoblast cells," Electrophoresis, vol. 25, no. 9, pp. 1342-1352, 2004

[16]. G. Drewes and T. Bouwmeester, "Global approaches to proteinprotein interactions," CurrentOpinion in Cell Biology, vol. 15,no. 2, pp. 199-205, 2003.

[17]. Vitorino, R., Lobo, M. J., Ferrer-Correira, A. J., Dubin, J. R., Tomer, K. B., Domingues, P. M. \& Amado, F. M. (2004)Identification of human whole saliva protein components using proteomics. Proteomics 4, 1109-1115.

[18]. Walz, A., Stuhler, K., Wattenberg, A., Hawranke, E., Meyer, H. E., Schmalz, G., Bluggel, M. \& Ruhl, S. (2006) Proteome analysis of glandular parotid and submandibular-sublingual saliva in comparison to whole human saliva by twodimensional gel electrophoresis. Proteomics 6, 1631-1639.

[19]. Hu JC, Yamakoshi Y, Yamakoshi F, Krebsbach PH, Simmer JP. (2005) proteomics and genetics of dental enamel. Cell Tissues Organs, 181(3-4): 219-31.

[20]. Hu S, Loo JA, Wong DT. (2007) Human saliva proteome analysis. Ann N Y Acad sci. 1098(mar); 323-9.

[21]. Y Fleissig, O Deutsch, E Reichenberg, M Redlich, B Zaks, A Palmon, DJ Aframian (2008). Different proteomic protein patterns in saliva of Sjögren's syndrome patients. Oral Diseases 15(1), 61-8.

[22]. Reichenberg E, Redlich M, Cancemi P, Zaks B, Pitaru S, Fontana S, Pucciminafra I, Palmon A (2005). Proteomic analysis of protein components in periodontal ligament fibroblasts. Journal of periodontology 76, 1645-53.

[23]. R. Kathariya andA. R. Pradeep, "Salivaryproteomicbiomarkers for oral diseases: a review of literature," American Overseas School of Rome, vol. 1, pp. 43-49, 2010.

[24]. Kathariya R, A R Pradeep. Salivary proteomic biomarkers for oral diseases: a review of literature. Archives of Oral Sciences \& Research. 2010;1(1):43-49.

[25]. Mandel ID. The diagnostic uses of saliva. J Oral Pathol Med 1990; 19:119-125.

[26]. Schenkels LC, Veerman EC, NieuwAmerongen AV. Biochemical composition of human saliva in relation to other mucosal fluids. Crit Rev Oral Biol Med. 1995; 6(2):161- 75.

[27]. Masakazu N, Slots J. Salivary enzymes Origin and relationship to periodontal disease. J Periodontal Res. 1983; 18(6):559-569.

[28]. Mazengo MC, Tenovuo J, Hausen H. Dental caries in relation to diet, saliva and cariogenic micro-organisms in Tanzanians of selected age groups. Community Dent Oral Epidemiol. 1996;24:169-74.

[29]. Sreedhar A ,Shobha Prakash, Sapna N., Santhosh Kumar. Proteomics - The New Era of Periodontics. Journal of Dental Sciences and Research. 2011 Vol. 2(2):1-5.

[30]. Socransky S S. Relationship of bacteria to the etiology of periodontal disease. Journal of Dental Research. 1970 ; 49 (2): $203-222$

[31]. Loesche W L, Lopatin D E, Giordano J, Alcoforado G, Hujoel P. Comparison of the benzoyl-DL-argininenaphthylamide (BANA) test, DNA probes, and immunological reagents for ability to detect anaerobic periodontal infections due to Porphyromonasgingivalis, Treponema denticola, and Bacteroidesforsythus. Journal of Clinical Microbiology. 1992; 30(2): 427-433.

[32]. Xia Q, Wang T, Taub F, Park Y, Capestancy CA, Lamont RJ, et al;. Quantitative proteomics of intracellular Porphyromonasgingivalis (2007). Proteomics.7 (23), 4323- 37.

[33]. Young-Jin Choi, Sun-HeeHeo, Jae-Mok Lee and Je-Yoel Cho, Identification of azurocidin as a potential biomarker by a proteomic analysis of gingival crevicular fluid. Proteome Science. 2011; 9:42.

[34]. Grant MM, Creese AJ, Barr G, Ling MR, Scott AE, Matthews JB, et al;. Proteomic analysis of a non-invasive human model of acute inflammation and its resolution: The twenty-one day gingivitis model. J Proteome Res 2010;9:4732-4744.

[35]. Hye Won Park, Jun-Seop Shin and Chan-Wha Kim (2007). Proteome of mesenchymal stem cells. Proteomics; 7, $2881-94$.

[36]. UCLA.Human Salivary Proteome Project Website. Available at: hspp.dent. ucla.edu/OFNASET.htm. Accessed Dec. 22, 2011.

[37]. Sreedhar A, Shobha P, Sapna N, Santhosh K. Proteomics - The New Periodontics.Journal of Dental Sciences and Research 2011; 2(2):1-5.

[38]. Giannobile William V, Beikler Thomas, Kinney Janet S, Ramseier Christoph A, Morelli Thiago, Wong David T. Saliva as a diagnostic tool for periodontal disease: current state andfuture directions. Periodontol. 2000;2009:52e64. 